\title{
Effect of Edaravone, a Free Radical Scavenger, on Ischemic Cerebral Edema Assessed by Magnetic Resonance Imaging
}

\author{
Satoshi SUDA, Hironaka IgARASHI*, Yasushi ARAI, Jyun ANDOU**, \\ Tetsuro CHISHIKI***, and Yasuo KATAYAMA
}

\begin{abstract}
Division of Neurology, Nephrology, and Rheumatology, Department of Internal Medicine, Nippon Medical School, Tokyo; *Center for Integrated Human Brain Science, Brain Research Institute, Niigata University, Niigata; Departments of

${ }^{* *}$ Radiology and ${ }^{* * *}$ Neurosurgery, Kitamurayama Hospital, Higashine, Yamagata
\end{abstract}

\begin{abstract}
The anti-edema effect of edaravone, a free radical scavenger, was evaluated by magnetic resonance imaging in six patients with extensive hemispheric ischemic stroke. $T_{2}$ relaxation time in the infarct core, the boundary zone of the infarct, and the noninfarcted hemisphere were calculated, and $T_{2}$ mapping was performed before and after edaravone administration. Edaravone administration significantly decreased the mean $T_{2}$ relaxation time in the boundary zone of the infarct from $121.5 \pm$ 9.2 (mean \pm standard deviation) to $114.5 \pm 9.9 \mathrm{msec}(p=0.008)$, but not in the core from $142.3 \pm 13.4$ to $142.2 \pm 18.5 \mathrm{msec}(p=0.97)$ or the noninfarcted hemisphere from $93.0 \pm 3.7$ to $93.1 \pm 3.8 \mathrm{msec}$ $(p=0.91)$. The $T_{2}$ subtraction map clearly demonstrated shortened $T_{2}$ relaxation time in the boundary zone of the infarct. The present results indicate that edaravone can salvage the boundary zone of the infarct and is a useful cytoprotective anti-edema agent.
\end{abstract}

Key words: edaravone, magnetic resonance imaging, free radical scavenger, ischemic cerebral edema, stroke

\section{Introduction}

Brain edema is one of the most important factors contributing to the morbidity and mortality associated with large hemispheric ischemic stroke, so effective anti-edema treatment in the acute phase is vitally important. Free radicals are believed to be important in the development of ischemic brain edema. ${ }^{4,5,19,22)}$ Edaravone (3-methyl-1-phenyl-2-pyrazolin-5-one) is the first free radical scavenger to have shown therapeutic effects in the treatment of acute ischemic stroke, and has been used clinically in Japan since 2001.6) Various cerebral ischemic models have demonstrated the protective effects of edaravone. Edaravone markedly attenuated ischemic brain edema in rats by preventing both nonenzymatic peroxidation and lipoxygenase activity. ${ }^{1)}$ Edaravone had protective effects against hydroperoxide-induced vascular endothelial injury in vitro, and attenuated vasogenic edema caused by

Received June 8, 2006; Accepted March 13, 2007 intracerebral microinjection of arachidonic acid in rats. ${ }^{16,23,24)}$ Moreover, edaravone showed antioxidant properties as well as anti-inflammatory effects that reduced the induction of inducible nitric oxide synthase. ${ }^{26)}$ Edaravone is thought to protect against ischemic brain edema based upon such experimental findings, but no anti-edema effect has not yet been clearly identified in clinical studies.

$\mathrm{T}_{2}$-weighted magnetic resonance (MR) imaging can both visualize and quantify vasogenic edema, so provides a method to assess the efficacy of ischemic stroke therapies against edema. . $^{3,8,11,17,18)}$ Previously, we characterized the anti-edema effect of glycerol after extensive ischemic stroke by comparing $\mathrm{T}_{2}$-weighted MR images obtained before and 1 hour after completing glycerol infusion. ${ }^{18)}$

The present study examined the anti-edema effect of edaravone in patients with extensive hemisphere ischemic stroke by comparing the $\mathrm{T}_{2}$ relaxation time and $\mathrm{T}_{2}$ mapping before and after edaravone administration in the infarct core, the boundary zone of the infarct, and the noninfarcted hemisphere. 
Table 1 Clinical summary of six patients

\begin{tabular}{|c|c|c|c|c|c|c|}
\hline $\begin{array}{l}\text { Case } \\
\text { No. }\end{array}$ & $\begin{array}{l}\text { Age (yrs)/ } \\
\text { Sex }\end{array}$ & $\begin{array}{l}\text { Lesion } \\
\text { side }\end{array}$ & Comorbidities & $\begin{array}{l}\text { NIHSS score } \\
\text { on admission }\end{array}$ & $\begin{array}{l}\text { Time to treatment } \\
\text { with edaravone after } \\
\text { stroke onset (hrs) }\end{array}$ & $\begin{array}{l}\text { Time to MR } \\
\text { examinations after } \\
\text { stroke onset (days) }\end{array}$ \\
\hline 2 & $69 / \mathrm{M}$ & $\mathrm{rt}$ & $\mathrm{AF}, \mathrm{DM}$ & 15 & 3 & 2 \\
\hline 3 & $75 / \mathrm{M}$ & lt & AF, HT & 19 & 4 & 3 \\
\hline 4 & $82 / \mathrm{F}$ & lt & $\mathrm{AF}, \mathrm{HL}$ & 21 & 5 & 3 \\
\hline
\end{tabular}

AF: atrial fibrillation, DM: diabetes mellitus, HL: hyperlipidemia, HT: hypertension, MR: magnetic resonance, NIHSS: National Institutes of Health Stroke Scale.

\section{Methods}

This study included six consecutive patients, four males and two females aged 67 to 82 years (mean \pm standard deviation $75 \pm 5.8$ years) with middle cerebral artery territory infarction who were admitted to the Neurology Department of the Kitamurayama Hospital between December 2001 and March 2002. The inclusion criteria to ensure informative automatic analysis with co-registration of images were: first stroke onset, nonhemorrhagic infarct, and absence of periventricular areas with marked high $\mathrm{T}_{2}$ signal intensity that could be interpreted as cerebral infarction. All six patients were treated under diagnoses of cardiogenic embolic infarction. Clinical data for these six patients are summarized in Table 1.

The basic treatment for the first 5 days from admission was continuous intravenous infusion of 10,000 units of heparin per day, intravenous drip infusion of $30 \mathrm{mg}$ of edaravone diluted with $100 \mathrm{ml}$ of saline given over 30 minutes twice daily, and 200 $\mathrm{ml}$ of $10 \%$ glycerol infused over 2 hours three times daily. Use of fibrinolytic and antiplatelet agents was avoided. All MR images were obtained 12 hours or more after the most recent glycerol injection, since the effect of glycerol is reported to persist for 4 to 6 hours. ${ }^{14,15)}$

MR imaging was performed at 2 to 4 days (mean 3 days) after symptom onset using a 1.5-T MR system (Visart; Toshiba, Tokyo). Plasma edaravone concentrations after intravenous infusion rapidly reach the maximum at 30 minutes, and then decrease rapidly. ${ }^{20,25)}$ Therefore, MR imaging was performed before and 1 hour after completion of edaravone infusion. Conventional spin-echo pulse sequences were used to calculate the $\mathrm{T}_{2}$ relaxation times, with other imaging conditions as follows: repetition time (TR) $4000 \mathrm{msec}$, echo time (TE) 30 or $100 \mathrm{msec}$ for the $\mathrm{T}_{2}$ map; or TR $300 \mathrm{msec}$ and TE 30 msec for $\mathrm{T}_{1}$-weighted images; field of view $256 \times$ $256 \mathrm{~mm}$; matrix size $256 \times 256$ pixels; and slice thickness $5 \mathrm{~mm}$ with no gap to obtain 20 contiguous slices. The recorded images were transferred to a workstation (Sparc 20; Sun, Milpitas, Calif., U.S.A.).

Segmentation of infarcted brain, noninfarcted brain, and ventricle was carried out automatically to avoid biasing the data. First, regions representing infarcted brain, ventricle, and normal brain were identified semiautomatically in the pretreatment $\mathrm{T}_{2}$-weighted MR image. The signal intensity for each pixel was determined using image-processing software (MR Vision, Menlo Park, Calif., U.S.A.) and transferred offline to a personal computer. Discriminant analysis was then performed using a statistics program (SPSS Inc., Chicago, Ill., U.S.A.) to calculate the threshold value of pixel intensity for reliably distinguishing infarcted brain and ventricle from normal brain on $\mathrm{T}_{2}$-weighted images. The threshold value of pixel intensity was calculated similarly for distinguishing ventricle from brain tissues on $\mathrm{T}_{1}$-weighted images. Segmentation of MR images into infarcted, noninfarcted, and ventricle could then be performed automatically using these threshold values as in our previous study (Fig. 1). ${ }^{18)}$

Pre- and posttreatment $\mathrm{T}_{2}$ maps were constructed from the TR/TE $=4000 / 30$ or $1000 \mathrm{msec}$ images. Three regions of interest, noninfarcted hemisphere, infarct boundary zone (inner $10 \mathrm{~mm}$ margin of the infarct), and infarct core, were localized in the preand posttreatment $\mathrm{T}_{2}$ maps (Fig. 1). Mean $\mathrm{T}_{2}$ relaxation time was calculated for each region from all slices. Finally the changes in $\mathrm{T}_{2}$ relaxation time were topographically visualized by co-registering the preand posttreatment $\mathrm{T}_{2}$ maps using imaging analysis software (MEDx 3.43; Medical Numerics, Inc., Sterling, Va., U.S.A.) according to image warping theory, and constructing the subtraction images. ${ }^{10)}$ MR images were evaluated by a neuroradiologist unaware of the clinical information. 


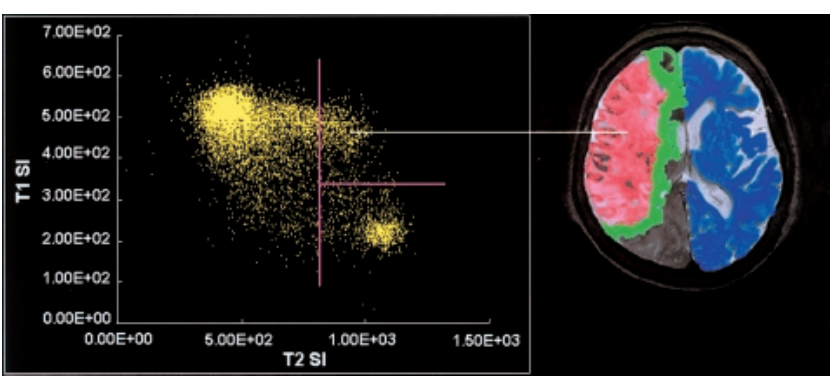

Fig. 1 Scatter plot of $T_{1}$ and $T_{2}$ signal intensities (SI). Vertical and horizontal lines show the thresholds determined by discriminant analysis. Segmentation of magnetic resonance images into infarcted area, noninfarcted area, and ventricle was carried out automatically using these thresholds. The upper right quadrant of the plot includes pixels in the infarcted area, identified at right. Three regions of interest were also defined on the pretreatment $T_{2}$ maps: blue area, noninfarcted hemisphere; green area, boundary zone of infarct; and red area, infarct core.
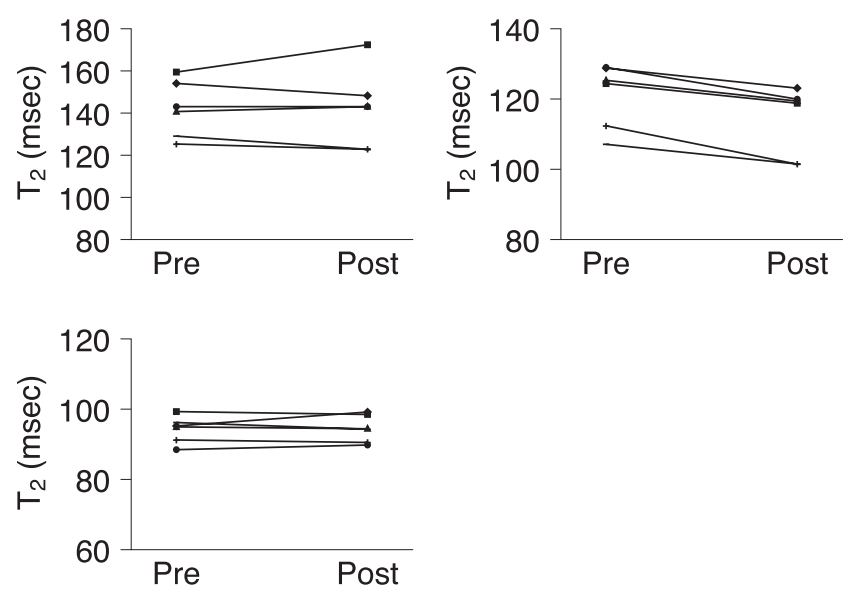

Fig. $2 T_{2}$ relaxation times before and after edaravone administration. $T_{2}$ relaxation time was shortened significantly in the boundary zone of the infarct after edaravone administration (upper right, $p=0.008$ ). No significant change was seen in the infarct core (upper left, $p=0.97$ ) or the noninfarcted hemisphere (lower, $p=0.91$ ).

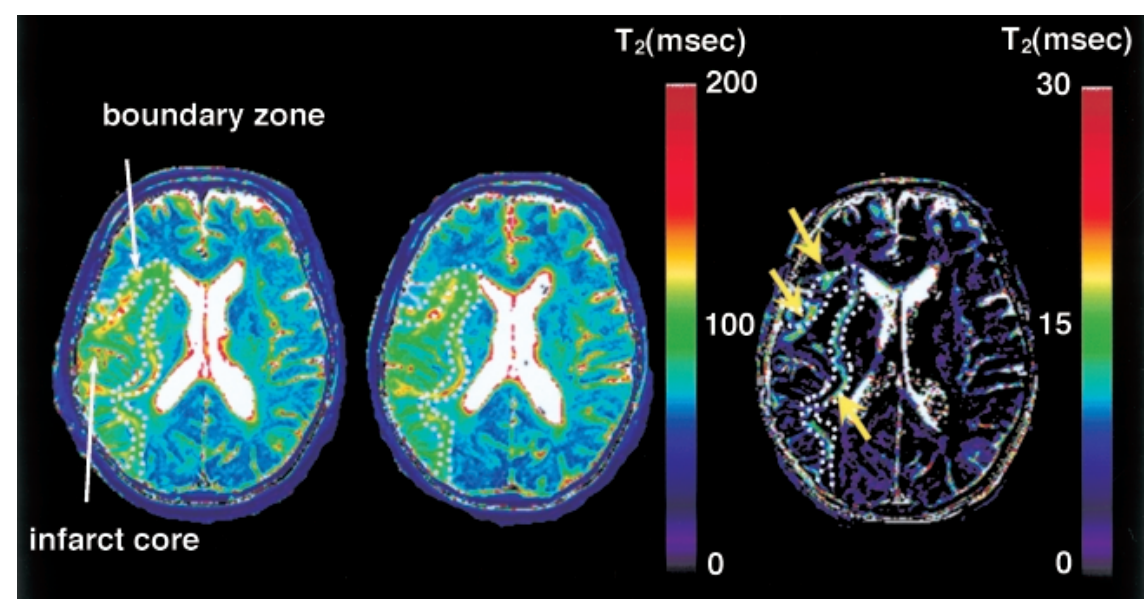

Fig. $3 \quad T_{2}$ maps before (left) and after edaravone administration (center), and $T_{2}$ subtraction map (right). The subtraction map clearly demonstrates shortened $T_{2}$ relaxation time in the boundary zone of the infarct (arrows).

The mean \pm standard deviation of the $\mathrm{T}_{2}$ relaxation times in the infarct core, the boundary zone of the infarct, and the noninfarcted hemisphere were compared before and after edaravone administration. The paired $t$ test was used for statistical analysis. A value of $\mathrm{p}$ below 0.05 was considered to indicate significance.

\section{Results}

$\mathrm{T}_{2}$ relaxation time declined significantly after edaravone administration in the boundary zone of the infarct from $121.5 \pm 9.2$ to $114.5 \pm 9.9 \mathrm{msec}$ ( $p=$ 0.008 ), whereas no significant change was evident in the infarct core from $142.3 \pm 13.4$ to $142.2 \pm 18.5$ msec $(p=0.97)$ or the noninfarcted hemisphere from $93.0 \pm 3.7$ to $93.1 \pm 3.8 \mathrm{msec} \quad(p=0.91)$ (Fig. 2). The $\mathrm{T}_{2}$ subtraction map clearly demonstrated shortened $\mathrm{T}_{2}$ relaxation time in the boundary zone of the infarct (Fig. 3).

\section{Discussion}

The present study of extensive ischemic cerebral infarcts using $\mathrm{T}_{2}$ relaxation time and $\mathrm{T}_{2}$ mapping demonstrated that treatment with edaravone was associated with decreased $\mathrm{T}_{2}$ relaxation time in the boundary zone, suggesting that this agent may 
attenuate ischemic cerebral edema.

In the present study, MR imaging was performed at mean 3 days after symptom onset. Ischemic brain edema in this phase consists mainly of vasogenic edema. ${ }^{13)}$ Overall mortality rate in patients with acute middle cerebral artery stroke ranges between $5 \%$ and $25 \%$, most often occurring within 1 week of onset in association with vasogenic edema. ${ }^{7,12)}$ Therefore, effective treatment of edema in the acute phase of extensive ischemic stroke is important, as well as thrombolytic, antiplatelet, and anticoagulant therapy.

Cerebral ischemic injury is accompanied by early dysfunction of the blood-brain barrier (BBB), subsequent vasogenic edema formation, and irreversible neuronal damage. Edaravone is thought to protect against ischemic brain edema mainly by inhibiting BBB injury. The present clinical study could not establish the pharmacologic action of edaravone in the clinical anti-edema effect. Further investigation of the molecular mechanisms of edaravone against ischemic cerebral edema is needed. However, the mechanism of the anti-edema effect of edaravone clearly differs from that of hyperosmotic therapy with glycerol or mannitol. Glycerol may decrease the water content of normal brain tissue, as $\mathrm{T}_{2}$ relaxation time of normal tissue decreased significantly after glycerol administration in a rat infarction model. Unfortunately, such changes in the uninvolved brain could aggravate shift of the midline structures, and actually exacerbate the mass effect of large cerebral infarct. ${ }^{8,9)}$

The present study showed that the $\mathrm{T}_{2}$ relaxation time declined significantly after edaravone administration only in the boundary zone of the infarct, not in the contralateral noninfarcted hemisphere, suggesting that edaravone acts only in the ischemic boundary zone of extensive ischemic stroke, and therefore should not exacerbate the mass effect. Treatment with edaravone also did not significantly shorten the $\mathrm{T}_{2}$ relaxation time in the infarct core. Previous experimental studies demonstrated that edaravone conferred neuroprotective effects only in the border zone. ${ }^{2,21)}$ The pharmacologic action of edaravone suggests that protective effects will only occur in ischemic territories with cells that are still viable.

Limitations of the present study include small sample size and absence of controls. Furthermore, the effect of edaravone against ischemic brain edema could be temporary rather than prolonged, since the time course of $\mathrm{T}_{2}$ relaxation time after treatment was not investigated. However, the present findings are consistent with those of previous experimental studies indicating that edaravone can salvage the boundary zone of the infarct, and is a promising cytoprotective anti-edema agent for the treatment of patients with ischemic stroke. Larger, placebo-controlled double-blind trials and investigation of the serial changes of $\mathrm{T}_{2}$ relaxation time will clarify the role of edaravone in the treatment of ischemic stroke.

\section{Acknowledgment}

This work was supported by a Grant-in-Aid for Scientific Research from the Ministry of Education, Culture, Sports Science and Technology of Japan.

\section{References}

1) Abe K, Yuki S, Kogure K: Strong attenuation of ischemic and postischemic brain edema in rats by a novel free radical scavenger. Stroke 19: 480-485, 1988

2) Amemiya S, Kamiya T, Nito C, Inaba T, Kato K, Ueda M, Shimazaki K, Katayama Y: Anti-apoptotic and neuroprotective effects of edaravone following transient focal ischemia in rats. Eur J Pharmacol 516: 125-130, 2005

3) Bacher A, Wei J, Grafe MR, Quast MJ, Zornow MH: Serial determinations of cerebral water content by magnetic resonance imaging after an infusion of hypertonic saline. Crit Care Med 26: 108-114, 1998

4) Chan PH: Role of oxidants in ischemic brain damage. Stroke 27: 1124-1129, 1996

5) Chan PH: Reactive oxygen radicals in signaling and damage in the ischemic brain. J Cereb Blood Flow Metab 21: 2-14, 2001

6) Edaravone Acute Infarction Study Group: Effect of a novel free radical scavenger, edaravone (MCI-186), on acute brain infarction. Randomized, placebocontrolled, double-blind study at multicenters. Cerebrovasc Dis 15: 222-229, 2003

7) Heinsius T, Bogousslavsky J, Van Melle G: Large infarcts in the middle cerebral artery territory. Etiology and outcome patterns. Neurology 50: 1940-1943, 1998

8) Izumi Y, Haida M, Kurita D, Sugiura T, Shinohara Y: [Evaluation of pharmacological efficacy of antiedema agents in a rat cerebral infarction model by MRI image analysis]. No Socchu 19: 117-124, 1997 (Jpn, with Eng abstract)

9) Izumi Y, Haida M, Kurita D, Sugiura T, Shinohara Y: [Evaluation of glycerol and mannitol by MRI image analysis: comparison between normal and ischemic brain in the rat]. Shinkei Chiryo 15: 389-394, 1998 (Jpn, with Eng abstract)

10) Jacobs MA, Windham JP, Soltanian-Zadeh H, Peck DJ, Knight RA: Registration and warping of magnetic resonance images to histological sections. Med Phys 26: 1568-1578, 1999

11) Kamada K, Houkin K, Iwasaki Y, Abe H: A high-field 
magnetic resonance imaging study of experimental vasogenic brain edema and its response to AVS: 1, 2-bis (nicotinamido)-propane. Acta Neurochir Suppl (Wien) 60: 491-493, 1994

12) Kaste M, Waltimo O: Prognosis of patients with middle cerebral artery occlusion. Stroke 7: 482-485, 1976

13) Klatzo I: Presidental address. Neuropathological aspects of brain edema. J Neuropathol Exp Neurol 26: 1-14, 1967

14) MacDonald JT, Uden DL: Intravenous glycerol and mannitol therapy in children with intracranial hypertension. Neurology 32: 437-440, 1982

15) Node Y, Nakazawa S: Clinical study of mannitol and glycerol on raised intracranial pressure and on their rebound phenomenon. Adv Neurol 52: 359-363, 1990

16) Ochi H, Morita I, Murota S: Mechanism for endothelial cell injury induced by 15-hydroperoxyeicosatetraenoic acid, an arachidonate lipoxygenase product. Biochim Biophys Acta 1136: 247-252, 1992

17) Qiao M, Malisza KL, Del Bigio MR, Tuor UI: Correlation of cerebral hypoxic-ischemic T2 changes with tissue alterations in water content and protein extravasation. Stroke 32: 958-963, 2001

18) Sakamaki M, Igarashi $H$, Nishiyama $Y$, Hagiwara $H$, Ando J, Chishiki T, Curran BC, Katayama Y: Effect of glycerol on ischemic cerebral edema assessed by magnetic resonance imaging. J Neurol Sci 209: 69-74, 2003

19) Schmidley JW: Free radicals in central nervous system ischemia. Stroke 21: 1086-1090, 1990

20) Shibata $H$, Arai S, Izawa M, Murasaki M, Takamatsu Y, Izawa O, Takahashi C, Tanaka M: [Phase I clinical study of MCI-186 (Edaravone, 3-methyl-1-phenyl-2pyrazolin-5-one) in healthy volunteers: Safety and pharmacokinetics of single and multiple administrations]. Rinsho Yakuri 29: 863-876, 1998 (Jpn, with Eng abstract)

21) Shichinohe H, Kuroda S, Yasuda H, Ishikawa T, Iwai M, Horiuchi M, Iwasaki Y: Neuroprotective effects of the free radical scavenger Edaravone (MCI-186) in mice permanent focal brain ischemia. Brain Res 1029: 200-206, 2004

22) Wahl M, Unterberg A, Baethmann A, Schilling L: Mediators of blood-brain barrier dysfunction and formation of vasogenic brain edema. J Cereb Blood Flow Metab 8: 621-634, 1988

23) Watanabe T, Egawa M: Effects of an antistroke agent MCl-186 on cerebral arachidonate cascade. J Pharmacol Exp Ther 271: 1624-1629, 1994

24) Watanabe T, Morita I, Nishi H, Murota S: Preventive effect of MCI-186 on 15-HPETE induced vascular endothelial cell injury in vitro. Prostaglandins Leukot Essent Fatty Acids 33: 81-87, 1988

25) Yokota S, Kumagai Y, Uchiumi M, Izawa S, Murasaki $M$, Akimoto $\mathrm{K}$, Iwamoto $\mathrm{M}$, Yuasa $\mathrm{T}$, Iwano M: [A pharmacokinetic study of MCI-186, a novel drug for cerebrovascular disease in elderly and young healthy subjects]. Rinsho Yakuri 28: 693-702, 1997 (Jpn, with Eng abstract)
26) Zhang N, Komine-Kobayashi M, Tanaka R, Liu M, Mizuno Y, Urabe T: Edaravone reduces early accumulation of oxidative products and sequential inflammatory responses after transient focal ischemia in mice brain. Stroke 36: 2220-2225, 2005

Address reprint requests to: Satoshi Suda, M.D., Division of Neurology, Nephrology, and Rheumatology, Department of Internal Medicine, Nippon Medical School, 1-1-5 Sendagi, Bunkyo-ku, Tokyo 113-8602, Japan.

e-mail: suda-sa@nms.ac.jp

\section{Commentary}

Edaravone has been demonstrated in a number of in vitro studies to diminish cerebral edema. This free radical scavenger is thought to protect against ischemic brain edema primarily by inhibiting bloodbrain barrier injury. In the current study, the authors have studied six selected patients with ischemic stroke to determine the effects of edaravone on ischemic cerebral edema as determined by $\mathrm{T}_{2}$ relaxation time on MRI. The patients utilized in the study had all experienced their first non-hemorrhagic stroke, with absence of periventricular involvement, and all were presumed to have cardiogenic embolic infarctions. The patients were treated with both edaravone and $10 \%$ glycerol. To eliminate any potential beneficial effects of glycerol on cerebral edema, the MR images were obtained at least twelve hours after glycerol administration, and before and one hour after the administration of edaravone. Segmentation of infarcted brain, noninfarcted brain to ventricle was carried out automatically to avoid bias in interpreting the data.

The authors found that the mean $\mathrm{T}_{2}$ relaxation time in the boundary zone of the infarct was decreased significantly following the administration of edaravone. No significant change occurred in the infarcted core or in the noninfarcted hemisphere after edaravone administration.

The demonstration that edaravone administration significantly decreases the $T_{2}$ relaxation time only in the boundary zone of an infarct and not in the contralateral noninfarcted hemisphere suggests that edaravone may be useful in combating cerebral edema following ischemic infarction. As pointed out by the authors, the current study includes a very small sample size and no controls. These findings, however, should stimulate larger scale investigations to better understand the potential role of edaravone in this setting. 
Daniel L. BARROW, M.D. Department of Neurosurgery Emory University Atlanta, Georgia, U.S.A.

Edaravone (3-methyl-1-phenyl-pyrazolin-5-one), a newly developed free radical scavenger, has been used in patients with acute brain infarction since April 2001 in Japan. Edaravone shows antioxidant effect through enhancement of prostacyclin production, inhibition of lipoxygenase metabolism of arachidonic acid by hydroxyl radicals, and eradication of active oxygen, leading to protection of endothelial cells against damage.

The present study evaluated the anti-edema effect of edaravone by calculating the $\mathrm{T}_{2}$ relaxation time in the infarct core, the boundary zone, and the noninfarcted hemisphere. The present results show significant decline in $\mathrm{T}_{2}$ relaxation time in the boundary zone but not in the infarct core or the noninfarcted hemisphere.

Although the present study demonstrated that edaravone is effective in reducing brain edema in the boundary zone of the infarcted area, it has limitations in demonstrating the actual clinical improvement in patients with edaravone administration. The cytoprotective effect of edaravone couldn't be sufficiently demonstrated by showing simple reduction in brain edema in the boundary zone of infracted area, but rather could be proved by showing reduction in the final infarcted volume on diffusion magnetic resonance imaging (MRI) or superiority in clinical outcome which are compared with patients who do not have edaravone treatment.

One more thing that should be kept in mind is that the complication of edaravone administration is not negligible, especially in elderly people. Acute renal failure, liver dysfunction, disseminated intravascular coagulation, and thrombocytopenia are observed more than $5 \%$ of patients who received edaravone treatment.

Notwithstanding the limitation of this study, the authors successfully demonstrated the efficacy of edaravone in reducing vasogenic edema in the boundary zone of the infarcted area using MRI $\mathrm{T}_{2}$ relaxation time. The result of this study will urge many neurosurgeons and neurologists to study the real impact of edaravone in the treatment of ischemic stroke.

Dae Hee Han, M.D. Department of Neurosurgery Seoul National University Hospital Seoul, R.O.K.

The authors examined the effect of edaravone, a free radical scavenger, on cerebral edema in six patients with middle cerebral artery occlusion due to cardiogenic embolism. MR imaging was performed before and 1 hour after completion of the edaravone treatment between 2 and 4 days after the onset. The $\mathrm{T}_{2}$ relaxation time in the infarct core, the boundary zone of the infarct, and the noninfarcted hemisphere on the MR images were calculated automatically. The results clearly showed that the $T_{2}$ relaxation time significantly declined only in the boundary zone of the infarct after edaravone treatment. They concluded that edaravone can salvage the boundary zone of infarct and is a useful cytoprotective anti-edema agent in cerebral ischemia.

The present study not only supports the previous notion that edaravone can prevent the brain edema following experimental and clinical cerebral infarct but demonstrates that the edaravone improved the pre-existed brain edema in the boundary zone of the infarct. However, the limitations of this study include small sample size, absence of controls, and a lack of feasible mechanism for the edema resolution. I look forward to their further studies to clarify the mechanism of ischemic neuronal damages through the investigation of the protective effect of edaravone on brain edema

Hiroyuki KINOUCHI, M.D. Department of Neurosurgery Interdisciplinary Graduate School of Medicine and Engineering University of Yamanashi Chuo, Yamanashi, Japan 